\title{
La compétence hypertextuelle en anglais de spécialité
}

\section{Claudine Gardey}

\section{(2) OpenEdition}

\section{Journals}

Édition électronique

URL : http://journals.openedition.org/asp/2895

DOI : 10.4000/asp.2895

ISSN : 2108-6354

\section{Éditeur}

Groupe d'étude et de recherche en anglais de spécialité

\section{Édition imprimée}

Date de publication : 1 décembre 1998

Pagination : 347-356

ISSN : 1246-8185

\section{Référence électronique}

Claudine Gardey, «La compétence hypertextuelle en anglais de spécialité », ASp [En ligne], 19-22 | 1998, mis en ligne le 16 avril 2012, consulté le 27 avril 2019. URL : http:// journals.openedition.org/asp/2895; DOI : 10.4000/asp.2895

Ce document a été généré automatiquement le 27 avril 2019

Tous droits réservés 


\title{
La compétence hypertextuelle en anglais de spécialité
}

\author{
Claudine Gardey
}

1 Le développement rapide des nouvelles technologies de l'information bouleverse les schémas traditionnels de communication, et l'étudiant que nous formons aujourd'hui devra, dans le monde professionnel de demain, faire la preuve de ses capacités à chercher, recevoir et transmettre des données par le biais de l'informatique : il devra donc posséder de véritables compétences dans le domaine de la lecture-compréhension de documents électroniques, aura appris à gérer avec efficacité l'hyperespace documentaire et saura créer des documents multimédias originaux, alliant texte, son et image.

2 Une certaine "euphorie technologique ", entretenue par les médias et les responsables politiques, nous amène à penser que tout utilisateur, guidé par son raisonnement intuitif, navigue à son gré dans l'univers des données numériques et établit facilement des réseaux de sens qui l'aident à progresser de manière ludique et naturelle.

3 L'observation montre que la réalité est assez éloignée de cette vision idyllique, car l'apprentissage électronique ne résout en rien les difficultés dues à une maitrise insuffisante des stratégies de lecture-compréhension-mémorisation. En fait, la présentation d'informations sous forme d'hypertextes constitue plus souvent un obstacle qu'un élément facilitateur pour l'utilisateur débutant.

4 Rappelons que l'on appelle hypertexte un ensemble de documents écrits, organisés en réseau non linéaire grâce à des liens sémantiques, les hypermots, que l'on active pour accéder à l'information choisie. Un document multimédia, ou plus précisément multimodal, est un ensemble de données transmises par des canaux perceptifs variés: texte, son, image fixe ou mobile. L'hypermédia est la conjonction de ces deux modes de présentation, soit un système non linéaire d'informations multimodales.

5 Ces distinctions, utiles au chercheur pour lever toute ambiguïté dans la transmission de son message, n'ont en fait que peu d'importance pour l'enseignant qui s'efforce avant tout d'aider l'apprenant à interagir rapidement et efficacement avec les données qu'il 
reçoit par l'intermédiaire de la machine. La "consultation interactive» de documents hypertextuels ou hypermédia requiert de la part de l'utilisateur une démarche cognitive différente de celle mise en oeuvre dans la lecture traditionnelle (Petit, 1998 : 1-14), et la maîtrise de ce nouveau processus de construction de sens exige un apprentissage préalable.

6 Un tel objectif présuppose une réflexion sur les problèmes spécifiques posés par ces modes de communication ainsi que l'identification des savoir-faire indispensables à la construction d'une véritable compétence hypertextuelle (electronic literacy skills). La prise en compte des différents paramètres débouche alors sur la conception de tâches d'apprentissage qui vont permettre à l'apprenant de découvrir par lui-même un certain nombre de stratégies efficaces et adaptées à ses propres orientations cognitives. Un dispositif de guidage de souplesse variable accompagne l'étudiant dans sa démarche heuristique.

\section{Lecture-compréhension en hypertexte}

\subsection{Spécificités de la lecture écran}

7 Tout utilisateur des nouvelles technologies conviendra qu'il est beaucoup plus difficile de lire un texte sur écran que sur document papier car les formats de présentation des pages-écrans sont très différents $d u$ format page imprimée et limitent le recours aux stratégies de lecture traditionnelles. C'est pourquoi le novice a tendance à imprimer tout le matériel qu'il rencontre sans procéder à la sélection réfléchie pratiquée par l'expert.

Volume-écran

8 La première étape de l'évaluation perceptive d'un document consiste en l'appréciation du volume des informations qu'il contient. En effet, les attentes cognitives s'organisent différemment suivant l'importance du document à étudier (thèse ou article de quelques pages par exemple) et déterminent les stratégies d'appréhension de l'information.

9 Contrairement au livre, l'hypertexte a une structure externe invisible et l'indication du nombre d'octets ne suffit pas à donner une idée concrète de son volume. Cette appréciation quantitative exige à l'écran une démarche volontaire qui oblige à une exploration rapide des pages qui constituent le document électronique. A-t-on accosté un îlot ou un continent? La plupart des étudiants n'ont pas spontanément des réflexes d'explorateur averti : ils restent prudemment près des côtes, à examiner de près les premières lignes sans chercher à prendre du recul vis-à-vis du document ni à comprendre son organisation globale; ils se plaignent ensuite de n'avoir pas eu le temps d'accéder à des informations intéressantes pendant le temps forcément limité de la leçon.

Espace-écran

10 Le deuxième obstacle de la lecture compréhension sur écran tient à l'espace visuel de lecture : un écran standard de 14 pouces, soit $35 \mathrm{~cm}$ en diagonale, restitue à peine plus du tiers d'une page papier, ce qui a pour effet de ralentir chez le lecteur la perception et la mise en relation des mots-clés qui permettent une reconstruction efficace du sens du document. Le fait d'additionner mentalement l'information contenue dans plusieurs écrans successifs exige un effort de mémorisation bien plus grand que lorsque la consultation s'effectue sur une page papier où les indices saillants sont immédiatement perceptibles (Brouste \& Cotte 1993 : 50-51). 
11 De plus, sur un certain nombre de sites, les journaux électroniques par exemple, l'organisation typographique d'un texte est simplifiée par rapport à celle d'un document imprimé : en raison des variations entre les divers formats de réception, la gamme des styles disponibles est réduite et les différences subtiles entre titre ou sous-titre, caractères gras ou italique, sont presque gommées : de courts paragraphes se succèdent de façon linéaire et il est impossible d'y percevoir une quelconque organisation hiérarchique. Le regard glisse sur l'uniformité scintillante de l'écran et le lecteur a du mal à orienter sa lecture autour d'un réseau de mots-clés qui faciliterait sa compréhension.

\subsection{Tâches d'entraînement à la lecture-écran}

12 Un article d'USA Today sur les objectifs de la mission spatiale "Neurolab" a servi de support pour une première sensibilisation des étudiants aux stratégies de lecture-écran. La première tâche consiste en un écrémage en temps limité du texte suivi d'une mise en commun des informations recueillies. Cette version électronique de skimming traditionnel déroute les étudiants : il semble qu'il y ait pour beaucoup une distance psychologique entre eux et l'ordinateur, un stress machinique à surmonter. En effet, tous ne possèdent pas le même degré de maîtrise dans la manipulation des curseurs et ont du mal à se concentrer sur la tâche ; d'autre part, en raison d'une mauvaise évaluation du volume du document, ils s'effraient de sa longueur (8 pages-écrans pour 3 pages imprimées), n'arrivent pas à en appréhender les limites et se sentent rapidement perdus.

La seconde tâche vise à faire dégager par le lecteur la macrostructure invisible de l'article : il lui est demandé de repérer les paragraphes qui fournissent les informations importantes (qui, quand, pourquoi, etc.) et d'en établir sur papier une représentation schématique ou, pour reprendre les termes de Jean-François Rouet (1997: 167), « une représentation propositionnelle condensée et hiérarchisée ». Ici, l'article comporte des séquences d'un ou deux paragraphes informatifs suivis par quelques lignes de commentaire appréciatif. Cette activité incite l'apprenant à établir un contact plus étroit avec le texte-écran, à classer mentalement les informations qu'il contient. L'appropriation du message est plus facile avec un article papier traditionnel où les titres et sous-titres guident la compréhension et où il est possible d'agir physiquement sur le support en surlignant ou reliant par des flèches les idées importantes. Le lecteur d'hypertexte doit acquérir de nouveaux réflexes et compenser son impuissance à intervenir directement sur le texte-écran par des attitudes mentales plus actives où l'attention sélective et la mémorisation visuelle sont davantage sollicitées.

14 La troisième tâche peut se définir comme une prise de notes électronique : la consigne est de sélectionner les phrases clés de l'article et de les copier-coller grâce au traitement de texte pour créer ainsi un résumé de longueur limitée, facile à imprimer et à évaluer. Aux interactions cognitives liées à la manipulation mentale du message écrit s'ajoutent ici d'autres types d'interactions: des interactions machiniques au cours desquelles l'utilisateur découvre les opérations à effectuer sur le clavier, mais également des interactions sociales puisque les étudiants qui maîtrisent déjà l'outil informatique aident spontanément leurs camarades moins expérimentés. Le compagnonnage cognitif entre pairs (Delacote 1996) qui s'institue alors favorise la verbalisation des comportements et l'échange de savoirs et savoir-faire. L'expert est ainsi conduit à expliciter (en languecible) des procédures qu'il a automatisées depuis longtemps, et le novice construit par l'action ses compétences. 
Ce premier exemple montre comment familiariser l'apprenant avec quelques stratégies de lecture d'un texte-écran. Le document cité plus haut apporte des informations de nature purement textuelle, et ne comporte donc aucun indice visuel susceptible de faciliter la compréhension. Or, les systèmes multimédias offrent le grand avantage pédagogique de pouvoir présenter des données selon des modalités sensorielles différentes et simultanées : les CD-ROM de type encyclopédique proposent de nombreuses animations visuelles, parfois enrichies d'un commentaire enregistré, qui reprennent, illustrent et complètent les explications écrites fournies dans un paragraphe adjacent. L'information est présentée de façon redondante et contiguë. Les recherches en psychologie cognitive (Meyer \& Anderson 1992) ont montré que le double codage, par des voies sensorielles différentes, facilitait le traitement en profondeur des informations et favorisait leur mémorisation. Encore faut-il que les étudiants aient développé des stratégies de lecture des documents visuels, ce qui n'est pas spontané chez beaucoup d'entre eux : ainsi, lors d'un travail sur une animation du CD-ROM Encarta 98 intitulée « Rock et Propulsion », ils avaient à définir le sens du mot « nozzle ». Presque tous se sont précipités sur le dictionnaire unilingue directement consultable sur l'écran et ont obtenu la définition suivante : "a spout on a hose from which a jet issues ", ce qui, on le conviendra, ne les a guère avancés. Ils ont totalement négligé l'aide visuelle offerte par l'animation où ce mot apparaissait en légende. Un travail systématique sur les animations des encyclopédies électroniques devrait augmenter leurs capacités à traiter les stimuli plurimodaux et à se construire des représentations mentales aussi bien verbales que visuelles.

\section{Gestion de l'hyperespace documentaire}

Le deuxième point de cette communication concerne la gestion de l'hyperespace documentaire. Un hyperespace est un système qui associe un nombre infini d'informations sur un mode non linéaire et non hiérarchique, de sorte qu'il est possible de passer instantanément d'une donnée à une autre sans que l'utilisateur soit vraiment conscient des liens logiques qui les unissent. En fait, le système possède sa logique propre, parfois fort éloignée de la logique humaine (certains parlent même de la logique fractale du réseau Internet), ce qui ne va pas sans poser des problèmes à l'utilisateur qui se sent très rapidement désorienté. C'est pourquoi il lui faut apprendre à gérer ce chaos apparent d'informations fragmentées et déstructurées et à maintenir une certaine cohérence au cours de ses recherches.

\section{Anticiper pour mieux gérer}

17 Anticiper, c'est prévoir les implications d'une action et adapter sa conduite en conséquence. Lors d'un travail de collecte d'informations sur Internet, le choix du motclé ou du site qui lancera la recherche est déterminant puisque, en fonction du point du réseau auquel il donne accès, la navigation sera aisée ou laborieuse. Imaginez entrer dans Londres soit par la voie express soit par une petite route de banlieue! De plus, la maitrise insuffisante de la langue étrangère va jalonner le parcours de l'apprenant d'obstacles imprévus: ainsi, lors d'un travail de recherche sur le réchauffement du climat, peu d'étudiants ont pensé à utiliser l'entrée "Global Warming", pourtant vue dans un document précédent. Beaucoup ont essayé «Temperature Rise », qui a livré nombre de sites en rapport avec la métallurgie ou la médecine. Quant à ceux qui, au mépris de la 
syntaxe anglaise, ont tapé « Rise Temperature », ils ont rapidement trouvé un site coquin où des infirmières virtuelles se proposaient de faire monter leur propre température!

Si l'erreur linguistique est parfois récompensée sur Internet (la fameuse logique fractale qui élève le marginal au rang de norme ?), elle peut être également source de difficultés réelles, qui mettent l'accent sur les problèmes de compréhension homme-interface « intelligente ». Nous sommes là bien loin de la simple incorrection biffée d'un trait rouge dans un exercice de grammaire, qui sanctionne davantage une maladresse linguistique qu'une réelle infraction au code communicationnel. La rétroaction immédiate de la machine amène peu à peu les étudiants à accepter l'idée d'une syntaxe spécifique où les connecteurs and, near ou or ont des fonctions rhétoriques si précises qu'elles déterminent le degré de réussite d'une recherche. Cependant, le transfert de cette compétence hypertextuelle particulière vers un domaine linguistique plus large ne semble pas s'effectuer de façon automatique, peut-être parce que l'activité (Petit 1998) n'est pas mémorisée en dehors de la mémoire de travail, le résultat seul étant stocké et le processus seulement transitoire.

\section{Mise en projet}

Pour trouver son chemin dans un système hypertextuel, il faut avoir un but bien défini et faire preuve de persévérance afin de garder à l'esprit ses objectifs sans se laisser aller à des dérives stériles. C'est là une tendance bien naturelle que de suivre ses penchants et son intuition, mais le fait de papillonner au gré des vents électroniques n'est pas la façon la plus efficace de mener à bien une recherche documentaire. Un moyen ludique d'inciter les apprenants à se mettre en projet et à faire preuve d'attention sélective en cours de lecture est d'organiser un jeu-quizz thématique opposant plusieurs équipes, qui doivent parcourir un hypertexte aussi efficacement que possible afin de répondre à une série de questions.

La collecte d'informations permet aux étudiants d'approfondir leurs connaissances sur le thème choisi et d'organiser mentalement une ébauche de représentation hiérarchisée du site étudié. L'élaboration graphique de cette représentation, ou « carte conceptuelle » fait appel aux capacités de mémorisation et d'organisation des apprenants et les incite à prendre du recul afin de transformer un ensemble d'informations déstructurées en un tout logique et cohérent.

\section{Utilisation des cartes conceptuelles}

La phase de sensibilisation décrite plus haut est suivie d'une phase d'entraînement au cours de laquelle l'étudiant doit faire la preuve de sa capacité à utiliser la carte ou le plan proposé dans la page d'accueil de la plupart des sites à organisation complexe. L'observation montre que l'apprenant n'a pas spontanément recours à ces outils de guidage et conserve sur écran les stratégies de lecture linéaire qu'il emploie face à un document imprimé, qui se révèlent souvent inadéquates face au support hypertextuel. Même en appliquant les techniques de lecture en diagonale, il faut plusieurs minutes pour parcourir la rubrique d'Encarta 98, intitulée "Space Exploration » qui occupe l'équivalent électronique d'une bonne vingtaine de pages imprimées, alors qu'il suffit de cliquer sur l'une des entrées de la table des matières («Outline ») présentée à gauche de l'écran pour accéder instantanément au paragraphe sélectionné, et corriger tout aussi rapidement son choix si on pense s'être fourvoyé.

La compréhension d'une carte exige rigueur et flexibilité : de la rigueur pour évaluer les connotations éventuelles d'un concept exprimé de façon succincte, et de la flexibilité 
pour réagir rapidement face aux aléas de la navigation. Une grande souplesse d'esprit est également nécessaire pour permettre à l'utilisateur de s'adapter à l'infinie variété des formats de présentation, qui vont de l'organigramme très hiérarchisé, linéaire et verbal, au schéma heuristique, global et imagé, qui s'adresse directement à l'hémisphère visuel et intuitif de notre cerveau. Suivant ses préférences cognitives, de type plutôt analytique ou globaliste, le consultant décodera plus facilement l'un ou l'autre de ces deux modes de représentation (Gardey 1996).

Ce dernier exemple met l'accent sur une autre difficulté que rencontre le lecteur habitué à consulter des tables de matières de type traditionnel : face à des concepts exprimés sous forme iconique, l'hypernavigateur doit apprendre à passer d'un mode de raisonnement analogique à un mode symbolique qui fait appel à un niveau d'abstraction plus important. S'il semble facile de concevoir qu'une flèche orientée dans un sens ou un autre permet d'accéder à la page suivante ou précédente, la compréhension d'un pictogramme à référence culturelle très marquée n'est ni intuitive, ni immédiate et dépend fortement du contexte dans lequel elle apparaît: ainsi, l'image d'une pomme, objet polysémique remarquable de la culture occidentale, ne sera décodée comme un symbole de l'enseignement primaire américain que si elle est accompagnée du célèbre mortier universitaire (Learning Resource Server, College of Education, University of Illinois).

Enfin, comme l'explique Denis Legros (1997), les utilisateurs qui ont de faibles capacités à traiter l'espace doivent mettre en jeu une grande partie de leurs ressources cognitives pour déchiffrer le matériel présenté visuellement et le mettre en mémoire, ce qui a pour effet de diminuer d'autant les ressources affectées à la compréhension du message proposé. De ce fait, chez les sujets peu habiles à traiter l'espace, les hésitations et les retours en arrière fréquents augmentent considérablement le temps consacré à la navigation proprement dite au détriment de celui alloué à la prise de connaissance des informations.

Il semble enfin indispensable que les lecteurs des nouvelles sources d'informations fassent preuve d'esprit critique et sachent prendre du recul vis-à-vis des données qui leur parviennent sur l'écran.

Leur fiabilité n'est en effet que trop rarement remise en question par les utilisateurs qui ont tendance à adopter le point de vue du premier site intéressant qu'ils rencontrent. Ils n'ont pas toujours conscience de la nature commerciale ou polémique de certains sites. L'étude par plusieurs groupes des différentes facettes d'un fait de société, telles la guerre d'Irak ou la monopolisation du marché informatique par Microsoft, apprend aux étudiants à faire la part entre information et propagande et les encourage à montrer plus de circonspection au cours des recherches ultérieures.

\section{Des savoir-faire aux savoirs}

Nous venons de voir comment il était possible de développer quelques stratégies opératoires à partir d'hypertextes en anglais, sur des thèmes proches des champs d'intérêt des étudiants. Ces derniers sont donc exposés à un environnement linguistique riche, varié et authentique, souvent présenté de manière attrayante et ludique. La plupart des étudiants sont très motivés par un travail sur ordinateur, mais en retiennent-ils quelque chose de plus que s'ils avaient travaillé sur documents papier et les systèmes hypertextes sont-ils réellement des éléments facilitateurs de l'apprentissage ? La question 
qui se pose est donc de comprendre dans quelle mesure la découverte de procédures s'accompagne d'une construction par l'apprenant de nouvelles connaissances linguistiques. Il n'est pas facile d'en juger en raison du manque de recul et du trop petit nombre de recherches portant sur l'utilisation de documents hypermédia authentiques en classe de langue. Les didacticiels du commerce ou les exerciseurs répondent à un besoin bien légitime de manipuler la langue objet d'étude, mais l'objectif prioritaire des enseignants du secteur LANSAD est de promouvoir l'anglais en temps que vecteur de communication et de formation professionnelle.

On trouve sur Internet des sites spécialement conçus pour permettre à l'apprenant d'enrichir son vocabulaire ou corriger sa prononciation: ainsi le site Impact Online propose une série de textes de difficulté variable où un double-clic sur un mot inconnu fait apparaître sa définition en anglais, accompagnée de quelques exemples d'utilisation en contexte et de sa prononciation enregistrée. C'est là un bon moyen de sensibiliser à un thème, de favoriser les acquisitions terminologiques ou de remédier à quelques défaillances phonologiques.

29 Il semble pourtant encore plus intéressant de faire concevoir par les étudiants euxmêmes des documents similaires, sous la forme de comptes-rendus de recherche offrant un complément écrit, sonore et pourquoi pas visuel à la compréhension. Ce type de production oblige ses créateurs à se concentrer à la fois sur les aspects formels et communicatifs de la langue, puisqu'ils doivent prendre en compte la polysémie des mots à définir, vérifier leur prononciation et choisir la représentation graphique la plus adaptée à la transmission de leur message. L'intégration de ces documents dans les pages Web du département apporte alors à ces activités de production une réelle dimension communicative.

Une autre façon de concilier enjeux linguistiques et professionnels réside dans l'utilisation des CD-ROM publicitaires, envoyés à titre gratuit aux enseignants de disciplines scientifiques par de nombreuses multinationales. Ces CD-ROM à visée commerciale atteignent un niveau de compétence didactique que pourraient leur envier nombre d'entreprises spécialisées dans l'industrie des langues! Ils sont souvent concis, précis et cohérents, et la bonne coordination entre informations verbales et illustrations explicatives facilite la compréhension du matériel présenté.

31 Nous avons ainsi détourné de sa fonction première le $C D$ de démonstration d'un oscilloscope Infinium produit par Hewlett Packard, et ce avec d'autant moins de scrupules que nos collègues du département Génie électrique comptent réellement sur la collaboration des enseignants de langue pour former les étudiants à la compréhension des cahiers des charges et autres documents techniques en anglais.

Au lancement de ce CD-ROM, l'étudiant peut choisir entre le mode « World» qui propose des vidéos accompagnées de leur script et le mode «US » où la vidéo est présentée seule. Cette option permet à chacun de choisir le niveau de difficulté le plus adapté à ses compétences linguistiques. Ensuite, l'utilisateur navigue à son gré ou suivant des instructions préétablies afin de découvrir les performances de l'appareil ou établir la liste de ses fonctions spécifiques. Il écoute les explications de l'expert tout en lisant le script affiché simultanément à l'écran, observe en direct les résultats des manipulations, reçoit des précisions complémentaires et peut assister à cette présentation autant de fois qu'il le souhaite. Des fiches techniques imprimables à la demande permettent des activités d'approfondissement ultérieures. 
el CD-ROM joue le rôle d'expert sur des sujets où les compétences du professeur de langue sont assez limitées. L'enseignant n'est plus le transmetteur de connaissances, mais il devient le facilitateur de l'apprentissage en fournissant à la demande des aides linguistiques ou méthodologiques. Toutefois, la qualité de cette navigation dépend surtout de la capacité de l'étudiant à établir des liens entre ses savoirs antérieurs et le matériel qui lui est présenté. L'interface informatique est le réservoir virtuel d'une masse infinie de données, elle permet des échanges interactifs entre l'utilisateur et le système, mais, comme le dit Jean-François Rouet :

les nouvelles technologies de l'information sont porteuses d'un potentiel important en matière d'accès et d'utilisation de l'information pédagogique. Mais pour exploiter ce potentiel efficacement, il faut développer une véritable ingénierie pédagogique, basée sur les modèles cognitifs de la compréhension et l'acquisition des connaissances. (1997: 178)

Quelles capacités cognitives sont-elles donc nécessaires pour apprendre grâce aux nouvelles technologies de l'information? Du sens critique, une attention sans faille, une perception fine des indices verbaux et non verbaux, un mode de réaction à la fois réflexif et intuitif, un esprit d'analyse et de synthèse permettant le traitement à la fois séquentiel et global de l'information... c'est-à-dire des performances largement supérieures à celles que nous reconnaissent les cogniticiens, et qui témoigneraient d'une utilisation extrême des capacités de notre cerveau !

Ne rêvons pas: même si des tests sur la population enfantine d'Amérique du Nord semblent indiquer que l'infléchissement technologique de la société favorise le développement d'aptitudes spécifiques (La Recherche, 1998), il est impossible d'affirmer que la machine rend l'homme plus intelligent. Mais l'interaction avec une interface « intelligente» rend certainement l'utilisateur plus actif, donc plus impliqué dans la construction de ses propres savoirs. En fait, la dynamique communicationnelle générée par les nouvelles technologies nous amène à faire évoluer nos modes habituels d'appropriation des connaissances et à acquérir des réflexes cognitifs originaux.

\section{BIBLIOGRAPHIE}

Brouste, Pierre et Dominique Cotte. 1993. Le multimédia, promesses et limites, Paris : ESF.

Delacote, Goéry. 1996. Savoir apprendre, les nouvelles méthodes. Paris : Éditions Odile Jacob.

Gardey, Claudine. 1996. «Interactions et construction du savoir en classe de langues ». Thèse de doctorat, Université Bordeaux 2.

Legros, Denis. 1997. « La construction des connaissances par le multimédia ». In J. Crinon, \& C. Gautellier, Apprendre avec le multimédia. Paris : Retz, 181-191.

Mayer, R.E. et R.B. Anderson. 1991. « The instructive animation: An experimental test of dualcoding hypothesis ». Journal of Educational Psychology 83, 484-490.

Neisser, Ulric. 1998. « Sommes-nous plus intelligents que nos grands-parents? ». La Recherche 309, 46-52.

ASp, $19-22$ | 2012 
Petit, Isabelle. 1998. «Consultation interactive et logique cognitive ». Degrés 92-93: Penser le multimedia 97-98, 1-14.

Rouet, Jean-François. 1997. « Le lecteur face à l'hypertexte ». In J. Crinon, \& C. Gautellier, Apprendre avec le multimédia. Paris : Retz,165-180.

\section{Références numériques}

Encarta 98 Encyclopedia. 1998. CD-ROM, Microsoft Corporation.

Impact Online Ressources éducatives <http://lrs.ed.uiuc.edu>

Infinium High performance, low-frustration oscilloscope. 1997. CD-ROM, Product Overview Version 1.0, Hewlett Packard Company.

USA Today : « Neurolab studies long-term space flights » http://www.usatoday.com/life/science/ space/lss 1901

\section{RÉSUMÉS}

Étant donné l'importance grandissante des nouvelles technologies de l'information, l'utilisateur doit développer une véritable compétence hypertextuelle, nouvelle en ce sens qu'elle requiert la mise en oeuvre de stratégies cognitives d'une autre nature que celles habituellement employées face à des documents imprimés. Diverses tâches de type recherche d'informations ou résolution de problème peuvent aider l'apprenant d'anglais à comprendre la structure des systèmes hypertextes et à construire savoirs et savoir-faire en interagissant avec l'interface numérique.

Given the ever-growing importance of new technologies, the user has to develop electronic literary skills, which means putting into practice new cognitive strategies, different in nature from those currently used in the reading of printed material. Varied information-finding or problem-solving tasks may help the learner to understand the structure of hypertextual systems and build his declarative and procedural knowledge by interacting with the digital interface.

\section{INDEX}

Mots-clés : compétence hypertextuelle, interaction, nouvelles technologies, stratégie cognitive Keywords : cognitive strategy, electronic literary skill, new technologies

\section{AUTEUR}

\section{CLAUDINE GARDEY}

Claudine Gardey est professeur agrégée d'anglais au département GEII de l'IUT A, Université de Bordeaux 1. Elle a soutenu en 1996 une thèse de doctorat nouveau régime intitulée «Interactions et construction du savoir en classe de langues ». Elle intervient dans le cadre de la préparation de l'épreuve sur dossier du CAPES d'anglais (Bordeaux 3) et participe aux travaux du laboratoire de recherche LABORAL (DLVP Bordeaux 2). Ses recherches portent sur les aspects cognitifs de l'enseignement-apprentissage, la didactique de l'anglais de spécialité et les nouvelles technologies. claudine.gardey@u-bordeaux1.fr 